\title{
Utilización de Instagram como una Herramienta Pedagógica para la Enseñanza de Morfología en Tiempos de COVID-19
}

\author{
Use of Instagram as a Pedagogical Tool for Teaching Morphology in Times of COVID-19
}

Ignacio Roa

\begin{abstract}
ROA, I. Utilización de Instagram como una herramienta pedagógica para la enseñanza de morfología en tiempos de COVID-19. Int. J. Morphol., 39(4):1063-1067, 2021.

RESUMEN: El año 2020 se declaró a la COVID-19 como pandemia, afectando a Chile en sus actividades comerciales, sociales y de educación, haciendo imposible la interacción presencial estudiante-docente en todas las universidades del país. Lo anterior, sumado al difícil momento económico y social que los estudiantes atraviesan, se torna necesario la búsqueda de estrategias que lo acerquen al docente para brindarles de manera más sencilla y rápida el material de estudio. Instagram resulta ser una herramienta de fácil uso y acceso para todos. El objetivo fue utilizar la plataforma de redes sociales Instagram como una herramienta útil de acercamiento a contenidos de Histología y Embriología, para estudiantes de la Universidad de Talca, Chile. Como metodología, se utilizó la plataforma Instagram, con una cuenta diseñada para entregar material docente a estudiantes de primer y segundo año de la Universidad de Talca, en las carreras de las Ciencias de la Salud, en las áreas de Histología y Embriología, consistentes en preguntas teóricas y prácticas, figuras rotuladas, caricaturas y esquemas diseñados y confeccionados de manera propia. Dicho material fue puesto diariamente en la plataforma según las materias que el estudiantado debería ver por calendario en los distintos módulos. Luego de transcurrido el primer semestre del se procedió a realizar una encuesta con el fin de evaluar el impacto de la iniciativa en los estudiantes. Éstos percibieron la iniciativa docente de manera positiva, con metodologías que incentivaron la participación e interacción académico-estudiante. Resaltaron la calidad del material y el acceso más rápido y en todo momento desde sus teléfonos móviles. Instagram viene a ser una buena herramienta coadyuvante a otras metodologías de entrega de material docente por vías formales institucionales, permitiendo a los estudiantes un fácil acceso, rápido y de bajo costo.
\end{abstract}

PALABRAS CLAVE: Instagram; Redes sociales; Educación; Morfología; COVID-19.

\section{INTRODUCCIÓN}

En diciembre de 2019, el mundo fue sacudido por la aparición de un nuevo coronavirus en la ciudad de Wuhan, China, (Zhu et al., 2020), el cual se expandió rápidamente por todo el mundo, siendo declarado por la Organización Mundial de la Salud como pandemia el 11 de marzo del 2020 (Organización Mundial de la Salud, 2020). Su alta tasa de infectividad y rápida propagación, obligó a los distintos gobiernos a implementar medidas sanitarias tendientes al distanciamiento físico y confinamientos de distinto tipo (Ministerio de Salud, 2020).

Por su parte, las universidades chilenas, establecieron la utilización de teletrabajo y con esto la suspensión de toda actividad presencial con el estudiantado. En este contexto la vía de comunicación con nuestros estudiantes han sido los canales virtuales, acompañado de la creación de Becas de Conectividad, con el fin de asegurar que todos los estudiantes, tengan un acceso de calidad y equidad de desarrollo de sus actividades académicas.

La vía formal de comunicación de entrega de material docente entre el estudiante y profesor, en la mayoría de las universidades, son a través de plataformas de enseñanza online tipo Moodle, en las cuales es posible realizar evaluaciones, chats y subir material tipo video e imágenes, entre otros. Dicha plataforma y su utilización muchas veces saturan el internet domiciliario de los estudiantes, esto acompañado de la alta utilización de datos móviles por parte del estudiantado con el fin conectarse a clases, talleres, prácticos y pruebas hace que sus servicios de internet en muchas ocasiones no den abasto. Debido a lo anterior, se hizo necesario implementar estrategias complementarias a las vías formales de comunicación, con el fin de hacer llegar a todos material formal y de calidad en todo momento. En Chile 
múltiples empresas de telecomunicación ofrecen planes con redes sociales gratis, permitiendo al estudiante, sin mayor gasto de sus datos móviles, acceder a Facebook, Instagram, Twitter o TikTok. Lo anterior, sumado al acercamiento de la nuevas generaciones a estas redes sociales, nos permiten contar con una perfecta herramienta para llegar a los estudiantes. Solo como dato, podemos señalar que TikTok fue la aplicación más descargada en todo el mundo en marzo de 2020, con más de 115,2 millones de instalaciones y que, a enero de 2020, la plataforma Instagram tiene mil millones de usuarios activos (Javid, 2020), lo que la hace una red social ideal, debido a que permite subir todo tipo de imágenes, videos, encuestas, esquemas, preguntas y respuestas.

El objetivo fue describir la plataforma de redes sociales Instagram como una herramienta útil de acercamiento a contenidos de Histología y Embriología, para estudiantes de las Ciencias de la Salud de la Universidad de Talca, Chile.

\section{MATERIAL Y MÉTODO}

Como metodología, se procedió a crear una cuenta en la plataforma Instagram (ignacioroa28) en el mes de mayo de 2020; dicha cuenta fue diseñada para entregar material docente a estudiantes de primer y segundo año de la Universidad de Talca, de las carreras de las Ciencias de la Salud, en las áreas de Histología y Embriología. Para implementar la cuenta se utilizó un celular Iphone 7 (Apple Inc, USA) y los programas Pixaloop (Lightricks Ltd. USA) para realizar imágenes animadas y la pizarra de la aplicación Webex Teams (Cisco, USA) para realizar las caricaturas y esquemas (Fig. 1).
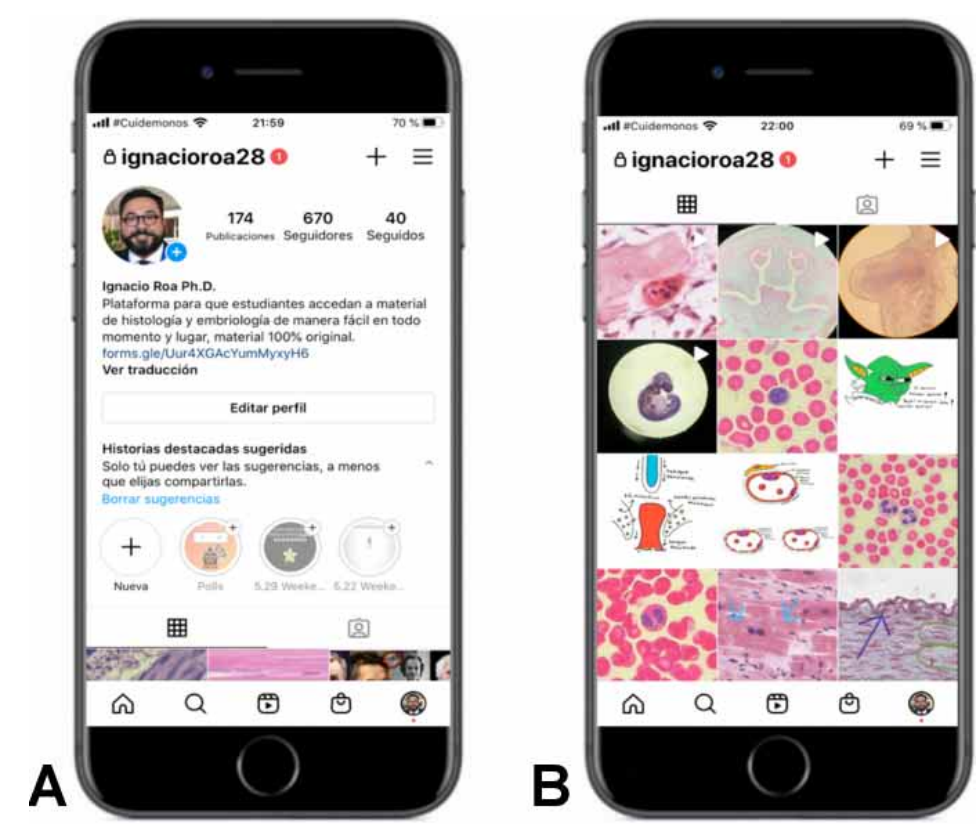

1064
El material consistió en preguntas teóricas y prácticas, figuras con y sin rótulos, caricaturas y esquemas diseñados y confeccionados de manera propia. Dicho material fue puesto en la plataforma diariamente, según las materias que el estudiantado debería ver por calendario en cada módulo.

Luego de transcurrido el primer semestre se procedió a realizar una encuesta en la extensión Drive de Google, con el objetivo de evaluar el impacto de la iniciativa en los estudiantes.

\section{RESULTADOS}

Un total de 153 estudiantes, todos de la Universidad de Talca, Chile, respondieron la encuesta. El $93 \%$ de los estudiantes había utilizado la cuenta ignacioroa28, para complementar su estudio. Los estudiantes que más hicieron uso de la plataforma cursaban las carreras de Odontología (26,4 $\%)$, Tecnología Médica (21\%) y Obstetricia y Puericultura $(21 \%)$. Hicieron uso de dicha plataforma por un periodo mayor a 4 meses $(64,1 \%)$ (Fig. 2).

Respecto a las preguntas ¿qué tanto le ha servido la plataforma? ¿qué tal le ha parecido el material entregado por dicha red social? ¿El Instagram contribuyó en su rendimiento durante las evaluaciones y pasos prácticos? gran porcentaje de los estudiantes contestó de manera positiva, indicando su utilidad tanto en sus evaluaciones como en sus pasos prácticos, así como la calidad del material entregado (Fig. 3). De acuerdo a la apreciación del estudiante con respecto al material entregado en la plataforma, el 51 $\%$ indicó que lo más útil fueron las imágenes de embriología, e imágenes de histología $(23,3 \%)$, además de las preguntas y respuestas $(9,6 \%$ ) (Fig. $4)$.

Fig. 1. A) Cuenta de Instagram de ignacioroa28 (B) con imágenes y videos representativos. 
¿En que Universidad estudias?

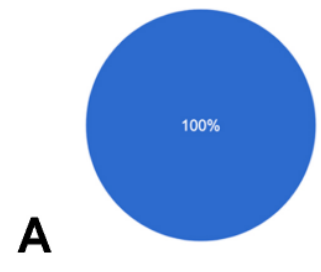

¿Qué carrera del área de la salud estudias?

B

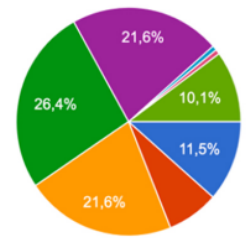

Haz utilizado el Instagram ignacioroa28 para estudiar Histologia/Embriologia
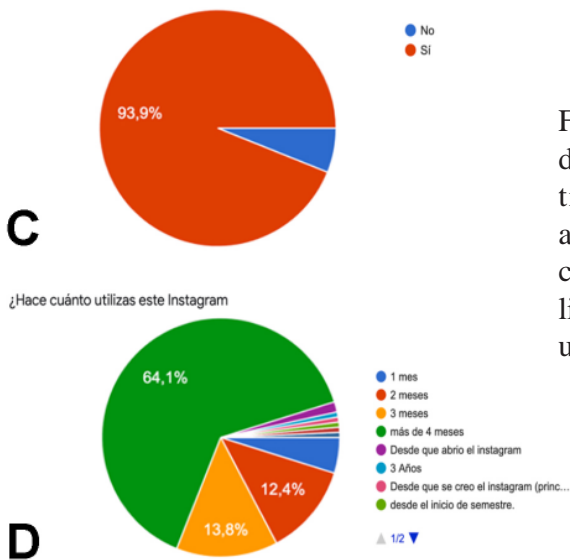

Fig. 2. Análisis de los resultados del Instagram ignacioroa28 mostrando (A) universidad del alumnado (B) carrera en la cual cursan sus ramos (C) nivel de utilización y (D) cantidad de meses utilizando la plataforma.
¿Que tanto te ha servido esta plataforma?

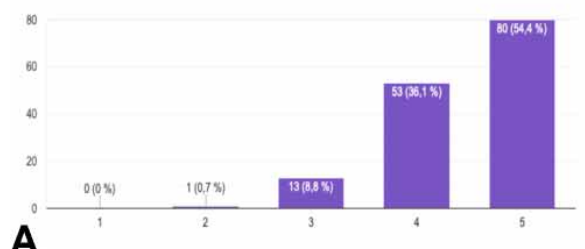

A

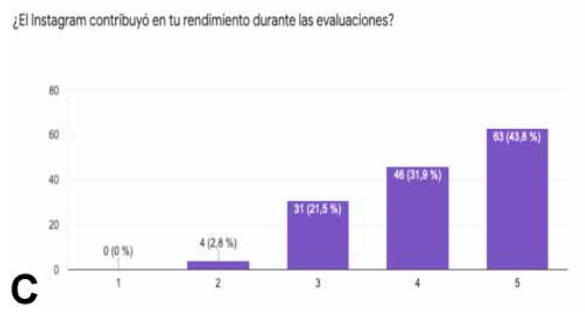

Que tal te ha parecido el material entregado en dicha red social?

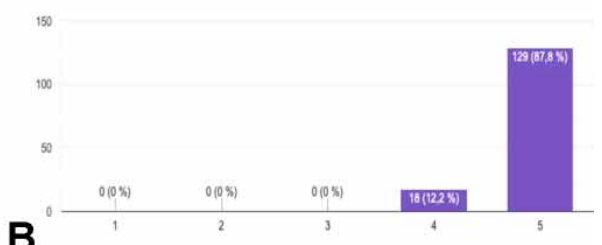

¿El Instagram contribuyó en tu rendimiento durante los pasos prácticos?

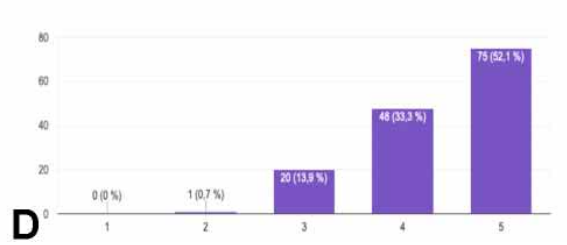

Fig. 3. Análisis de los resultados del Instagram ignacioroa28 mostrando $(\mathrm{A})$ nivel de satisfacción del alumnado con respecto a la totalidad de la plataforma (B) nivel de satisfacción del alumnado con respecto al material entregado (C) nivel de contribución de la plataforma a las evaluaciones y (D) a los pasos prácticos.

¿Qué material te ha parecido más importante para tu proceso educativo?

146 respuestas
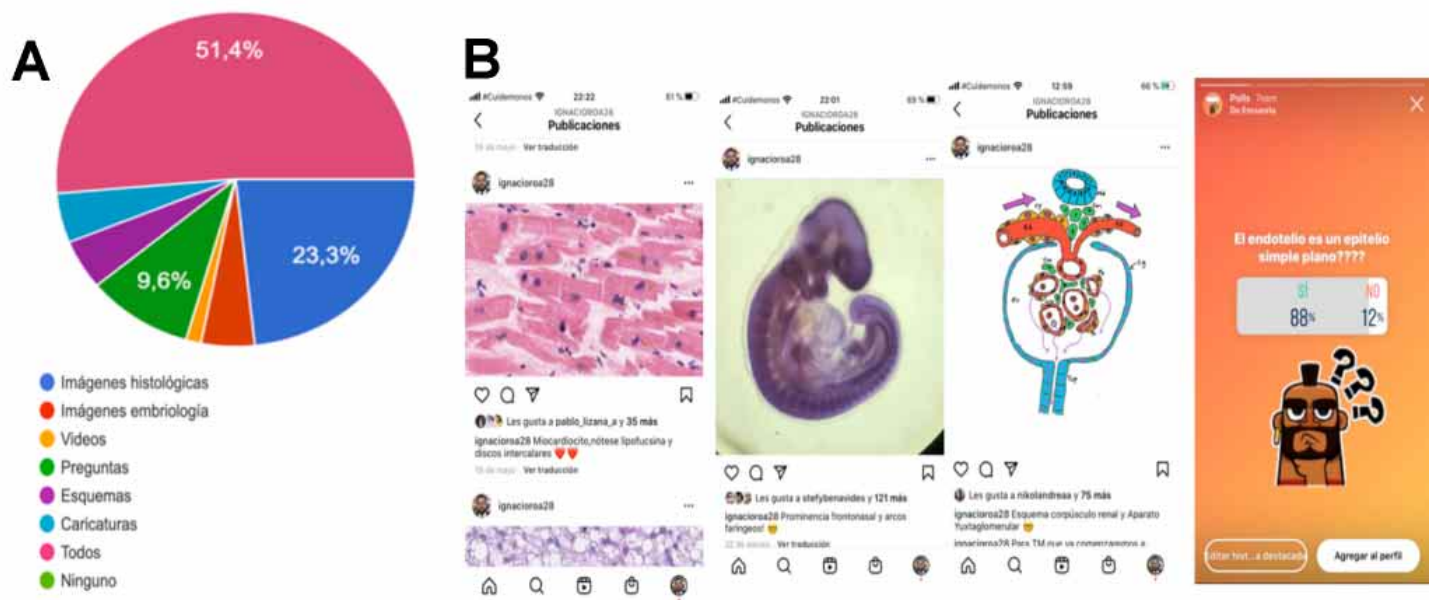

Fig. 4. Análisis de los resultados del Instagram ignacioroa28 mostrando (A) apreciación del tipo de material de estudio de la plataforma (B) ejemplo del material entregado en el Instagram ignacioroa28. 


\section{DISCUSIÓN}

Las redes sociales se componen de actividades que implican socializar y establecer contactos en línea a través de palabras, imágenes y videos. Facebook, Twitter, Path, Pinterest e Instagram son en su mayoría plataformas comunes de redes sociales conocidas por la gente (Handayani, 2015). Estas plataformas, tienen en la actualidad, un importante rol en la vida de los jóvenes (Handayani). En este ámbito, se encuentran los estudiantes quienes, día a día, utilizan estas plataformas con fines recreativos.

Instagram resulta ser una de las redes sociales más utilizadas a nivel mundial (Javid), en ella los usuarios comparten videos cortos y fotografías, las cuales pueden ser acompañadas con hasta 2.200 caracteres (Carpenter et al., 2020). De esta manera, es una herramienta a elección en las disciplinas de histología y embriología, en las cuales, figuras, esquemas y videos son vitales para el desarrollo de los aprendizajes comprometidos por nuestros syllabus. Estos syllabus plantean principalmente como objetivos: describir la composición, organización, procesos y estructuras del cuerpo humano, estableciendo relaciones morfofuncionales que existen entre las ciencias morfológicas (anatomía, embriología e histología), que explican posteriormente el desarrollo y estructura del ser humano en órganos, sistemas y en su constitución topográfica, permitiendo al estudiante aplicar estos conocimientos en la clínica.

Instagram, permite publicar videos, figuras, esquemas y preguntas-respuestas de manera sencilla (Shafer et al., 2018). Además, mensajes, publicaciones e historias entre usuarios los cuales pueden variar en cuanto a privacidad y formalidad (Carpenter et al.), esto último permite generar discusión de diversos temas, así como realizar preguntas privadas o públicas.

Los estudiantes pueden usar las redes sociales para mantenerse al día con sus amigos, con noticias y eventos actuales, ocupar el tiempo libre, encontrar contenido entretenido y compartir opiniones. Esto acompañado del fácil acceso y masificación del uso de equipos celulares, así como de planes con redes sociales gratuitas, hacen que estas se conviertan en una herramienta muy eficaz para comunicar e intercambiar ideas.

A través de Instagram los docentes podemos publicar fotos o videos que pueden ser comentados por sus seguidores, lo que permite desarrollar actividades en el aula de forma creativa. Debido a lo anterior, son numerosas las disciplinas que se encuentran utilizando Instagram con el fin de entregar material docente (Shafer et al.; Park et al.,
2018; Zhou \& Bercovitch, 2018). En nuestro caso, el material entregado así como la iniciativa tuvo gran aceptación de parte del estudiantado, debido principalmente a la rapidez de comunicación, el tipo de material factible de publicar y la posibilidad de comunicarnos en su "lenguaje". Los resultados indicaron un uso prolongado de la plataforma por la gran mayoría de las carreras de las Ciencias de la Salud de la Universidad de Talca, lo que de acuerdo a los encuestados, contribuyó a mejorar su comprensión durante las actividades prácticas, mejorando de esta manera sus calificaciones. Fue el material de embriología (imágenes reales de embriones en distintos estadios de desarrollo) el más valorado, seguido por las imágenes de histología y la sección de preguntas, estas últimas según los estudiantes muy valiosas, ya que le permitieron medir sus conocimientos y proceso de aprendizaje.

Podemos señalar que Instagram tiene un efecto beneficioso en los estudiantes, ayudándolos a entender de mejor manera los preparados histo-embriológicos. Es una herramienta educativa útil que permite brindar a estudiantes y docentes una manera fácil de comunicación que va más allá del horario de oficina y el aula. El uso de Instagram permite a los estudiantes generar ideas con contenido relevante al contexto y les ofrece una experiencia de aprendizaje que disfrutan. Finalmente, se puede enfatizar que el uso de Instagram en el aprendizaje de histología y embriología puede ser una herramienta innovadora y efectiva, si es utilizado de manera adecuada por estudiantes y profesores para fines educativos. Tiene como limitaciones el aprendizaje pasivo por parte del estudiantado y el requisito de personal comprometido para supervisar su uso, así como la imposibilidad de reemplazar la educación práctica en anatomía y sus ciencias afines, además de no poder evaluarse su impacto total en el aprendizaje (Douglas et al., 2019).

ROA. I. Use of Instagram as a pedagogical tool for teaching morphology in times of COVID-19. Int. J. Morphol., 39(4):1063$1067,2021$.

SUMMARY: In 2020, COVID-19 was declared a pandemic, affecting our country both in commercial, social and educational activities; making student-teacher interaction impossible in all the country's universities. This, added to the difficult economic and social times that our students are going through, make it increasingly necessary to search for strategies that bring us closer to providing them with study material in a simpler way. This is why Instagram turns out to be a tool that is accessible and easy to use. The objective was to describe the social media platform Instagram as a useful tool for accessing Histology and Embryology content, for students at the Universidad de Talca, Chile. As a methodology, the Instagram platform was used, with 
an account designed to deliver teaching material to first and second year students of the Universidad de Talca, in the Health Sciences degree programs, in areas of Histology and Embryology, consisting of theoretical and practical questions, labeled figures, cartoons and diagrams designed and created themselves. This material was posted on the platform daily according to the subjects that the students should see according to the calendar in the different modules. After the first semester of this year, a survey was taken in order to evaluate the impact of the initiative on students. The students perceived the teaching initiative in a positive way, with methodologies that encouraged academic-student participation and interaction. They highlighted the quality of the material and the quicker access at all times from their mobile phones. Instagram becomes a good tool to help other methodologies to delivery of teaching material through formal institutional channels, allowing students to access faster and at a low cost of navigation.

KEY WORDS: Instagram; Social network; Education; Morphology; COVID-19.

\section{REFERENCIAS BIBLIOGRÁFICAS}

Carpenter, J. P.; Morrison, S. A.; Craft, M. \&Lee, M. How and why are educators using Instagram? Teach. Teach. Educ., 96:103149, 2020.

Douglas, N. K. M.; Scholz, M.; Myers, M. A.; Rae, S. M.; Elmansouri, A.; Hall, S. \& Border, S. Reviewing the role of Instagram in education: Can a photo sharing application deliver benefits to medical and dental anatomy education? Med. Sci. Educ., 29:1117-28, 2019.

Handayani, F. Instagram as a teaching tool? Really? Proceedings of ISELT FBS Universitas Negeri Padang, 4(1):320-7, 2015.

Javid, M. Is it time to acknowledge Instagram as a home to medical education? Linkedin, 2020. Disponible en: https://www.linkedin.com/ pulse/time-acknowledge-instagram-home-medical-education-michaeljavid-do?trk=related_artice_Is \%20it \%20time \%20to \%20acknowledge $\% 20$ Instagram \%20as \%20a \%20home \%20to \%20medical $\% 20$ education \%3F_article-card_title

Ministerio de Salud (MINSAL). Plan de Acción Coronavirus COVID-19. Santiago de Chile, Ministerio de Salud, Gobierno de Chile, 2020. Disponible en: https://www.minsal.cl/nuevo-coronavirus-2019-ncov/

Organización Mundial de la Salud. WHO Director-General's Remarks at the Media Briefing on 2019-nCoV on 11 February 2020. Ginebra, Organización Mundial de la Salud, 2020. Disponible en: https:// www.who.int/director-general/speeches/detail/who-director-general-sremarks-at-the-media-briefing-on-2019-ncov-on-11-february-2020

Park, J. H.; Christman, M. P.; Linos, E. \& Rieder, E. A. Dermatology on Instagram: An analysis of hashtags. J. Drugs Dermatol., 17(4):482-4, 2018.

Shafer, S.; Johnson, M. B.; Thomas, R. B.; Johnson, P. T. \& Fishman, E. K. Instagram as a vehicle for education: What radiology educators need to know. Acad. Radiol., 25(6):819-22, 2018.

Zhou, J. \& Bercovitch, L. Instagram and the dermatologist: An ethical analysis. J. Am. Acad. Dermatol., 78(6):1226-8, 2018.

Zhu, N.; Zhang, D.; Wang, W.; Li, X.; Yang, B.; Song, J.; Zhao, X.; Huang, B.; Shi, W.; Lu, R.; et al. A novel coronavirus from patients with pneumonia in China, 2019. N. Engl. J. Med., 382(8):727-33, 2020.

\author{
Dirección para correspondencia: \\ Dr. Ignacio Roa Henríquez \\ Unidad de Morfología \\ Departamento de Ciencias Básicas Biomédicas \\ Facultad de Ciencias de la Salud \\ Universidad de Talca \\ Lircay Av. s / n \\ Talca \\ CHILE
}

E-mail: iroa@utalca.cl

Recibido : 01-04-2021

Aceptado: 02-05-2021 